\title{
New Solar Telescope Observations of Magnetic Reconnection Occurring in the Chromosphere of the Quiet Sun
}

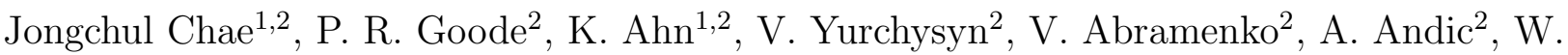 \\ $\mathrm{CaO}^{2}$, Y. D. Park ${ }^{3}$
}

\begin{abstract}
Magnetic reconnection is a process in which field-line connectivity changes in a magnetized plasma. On the solar surface, it often occurs with the cancellation of two magnetic fragments of opposite polarity. Using the 1.6 meter New Solar Telescope, we observed the morphology and dynamics of plasma visible in the $\mathrm{H} \alpha$ line, which is associated with a cancelling magnetic feature in the quiet Sun. The region can be divided into four magnetic domains: two pre-reconnection and two post-reconnection. In one post-reconnection domain, a small cloud erupted, with a plane-of-sky speed of 10 kilometers per second, while in the other one, brightening began at points and then tiny bright loops appeared and subsequently shrank. These features support the notion that magnetic reconnection taking place in the chromosphere is responsible for canceling magnetic features.
\end{abstract}

Subject headings: Sun: magnetic topology — Sun: chromosphere — Sun: photosphere - magnetic reconnection - magnetohydrodynamics

\section{INTRODUCTION}

Magnetic reconnection is believed to be at the heart of a variety of solar magnetic activities and the heating of the chromosphere and corona of the Sun, because it efficiently converts magnetic energy into thermal and kinetic energy of plasma and the relativistic kinetic energy of particles. Even though magnetic reconnection, in principle, can occur in the photosphere (Litvinenko 1999) and in the chromosphere (Chae et al. 2002a) as well as in the corona, the physical properties of magnetic reconnection occurring in the low atmosphere may be

\footnotetext{
${ }^{1}$ Astronomy Program, Department of Physics and Astronomy, Seoul National University, Seoul 151-747, Korea

${ }^{2}$ Big Bear Solar Observatory, 40386 North Shore Lane, Big Bear City, CA 92314,USA

${ }^{3}$ Korea Astronomy and Space Science Institute, 838 Daedeokdaero, Daejeon 305-348, Korea
} 
much different from that in the corona (Litvinenko \& Chae 2009). A well-known signature of magnetic reconnection in the low atmosphere is cancelling magnetic features (CMF), the collision and cancellation of two magnetic fragments of opposite polarity (Livi et al. 1985; Martin et al. 1985). A number of previous studies supported the notion that a CMF may be a manifestation of submergence of magnetic flux resulting from magnetic reconnection in the photosphere or in the chromosphere (Wang \& Shi 1993; Harvey et al. 1999; Chae et al. 2004; Kubo \& Shimizu 2007). The point is that a CMF does not represents the process of magnetic reconnection, but is likely to be its consequence. To reveal the physical relationship between a CMF and its associated magnetic reconnection, it is indispensable to observe the process of magnetic reconnection itself, as well as to observe the CMF at the same time.

How can one observe magnetic reconnection in the low atmosphere? Since we cannot directly measure magnetic field in the region of magnetic reconnection yet, if not on the solar surface, we have to observe plasma being involved in the reconnection. Commonly adopted observational manifestations of magnetic reconnection are brightenings and jet-like motions of plasma that are probably the outcome of heating and acceleration that proceed during the process of magnetic reconnection. These features, however, are not conclusive, since we still can not fully exclude the possibility that they may result from a sudden heating of other origins as well. More convincing observational evidence would be the identification of field-line connectivity, and its temporal change, which is the intrinsic property of magnetic reconnection (Priest \& Forbes 2000).

Despite a long history of observations, the $\mathrm{H} \alpha$ line still remains the most useful window into the solar chromosphere, in particular for the study of field-line connectivity in the chromosphere, mainly because magnetic field lines in the chromosphere are well traceable with the elongated structures of cool plasma visible in $\mathrm{H} \alpha$, like fibrils and loops (Foukal 1971). If the reconnection that is supposed to result in a CMF does occur in the chromosphere, there is a good possibility that it may be observable through the $\mathrm{H} \alpha$ line. As a matter of fact, for the first time we observed without ambiguity an event of magnetic reconnection occurring in the chromosphere in association with a CMF, using the 1.6 meter New Solar Telescope (NST, Goode et al. 2003, 2010) recently constructed at Big Bear Solar Observatory.

\section{OBSERVATION AND DATA REDUCTION}

The NST is an off-axis Gregorian telescope of with a clear aperture of 1.6 meter. Its diffraction-limited resolution is $0.10^{\prime \prime}$ at $\mathrm{H} \alpha$. The early scientific observations were carried out since April, 2009. On 2009 August 26 during the long-lasting extremely quiet period of solar activity, we observed a small region of the quiet Sun near the disk center using the $\mathrm{H} \alpha$ 

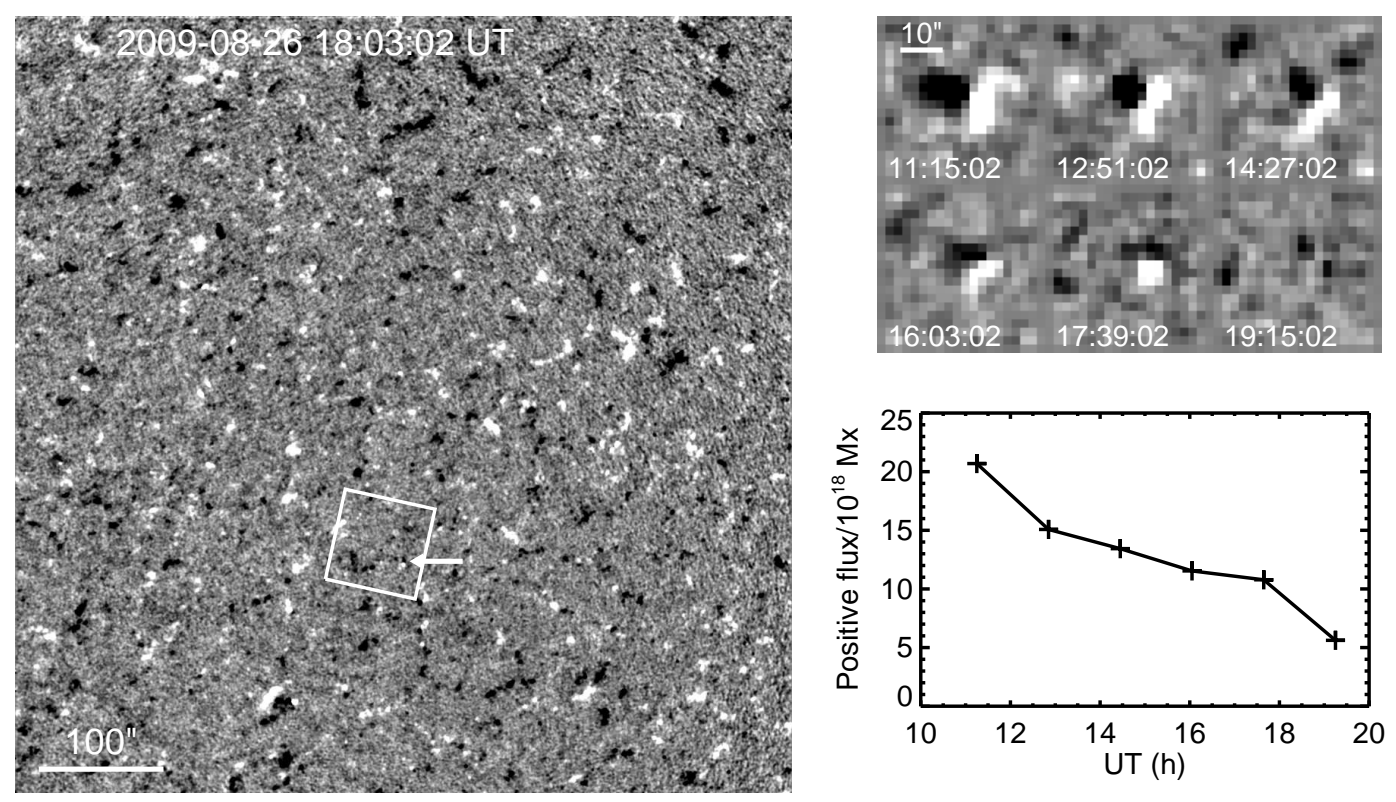

Fig. 1. - Left: CMF of our interest identified in a high-resolution magnetogram taken by SOHO/MDI. The square represents the field of view of NST observations, and the arrow points to the CMF. Right: the temporal variations of its morphology (top) and magnetic flux of positive polarity determined from the full-disk magnetograms of 96 minute cadence (bottom).

Lyot filter mounted on the Nasmyth bench of the NST. We chose the filter bandwidth of $0.5 \AA$ and tuned the central wavelength of the filter to the $\mathrm{H} \alpha$ line center. The images were recorded on a PCO CCD camera of $2048 \times 2048$ pixels. The duration of the observations of our interest was 12 minutes from 17:58:17 to 18:10:17 UT. During this period, we took a series of bursts each of which consisted of 100 raw images taken with a short exposure of $35 \mathrm{~ms}$. The cadence of bursts was $15 \mathrm{~s}$. After dark frame subtraction and flat fielding, the images were rebinned to a smaller size of $1024 \times 1024$ pixels to save computing time in data processing. The pixel size after the rebinning is $0.072^{\prime \prime}$. From each burst, we have obtained a high resolution image by applying Kiepenheuer-Institut Speckle Interferometry Package (KISIP) code (Wöger et al. 2008). The field of view effective after the speckle image construction is $70^{\prime \prime} \times 70^{\prime \prime}$. Every constructed image was aligned to its previous one with the technique of relative shift and destretching. In this study, we focus on a canceling magnetic feature in a small region of $22^{\prime \prime} \times 22^{\prime \prime}$.

We tracked some fine structures inside this region using the technique of non-linear affine velocity estimator (NAVE). This technique determines the displacement by non-linearly fitting two successive images to a model of image mapping based on the assumption that 


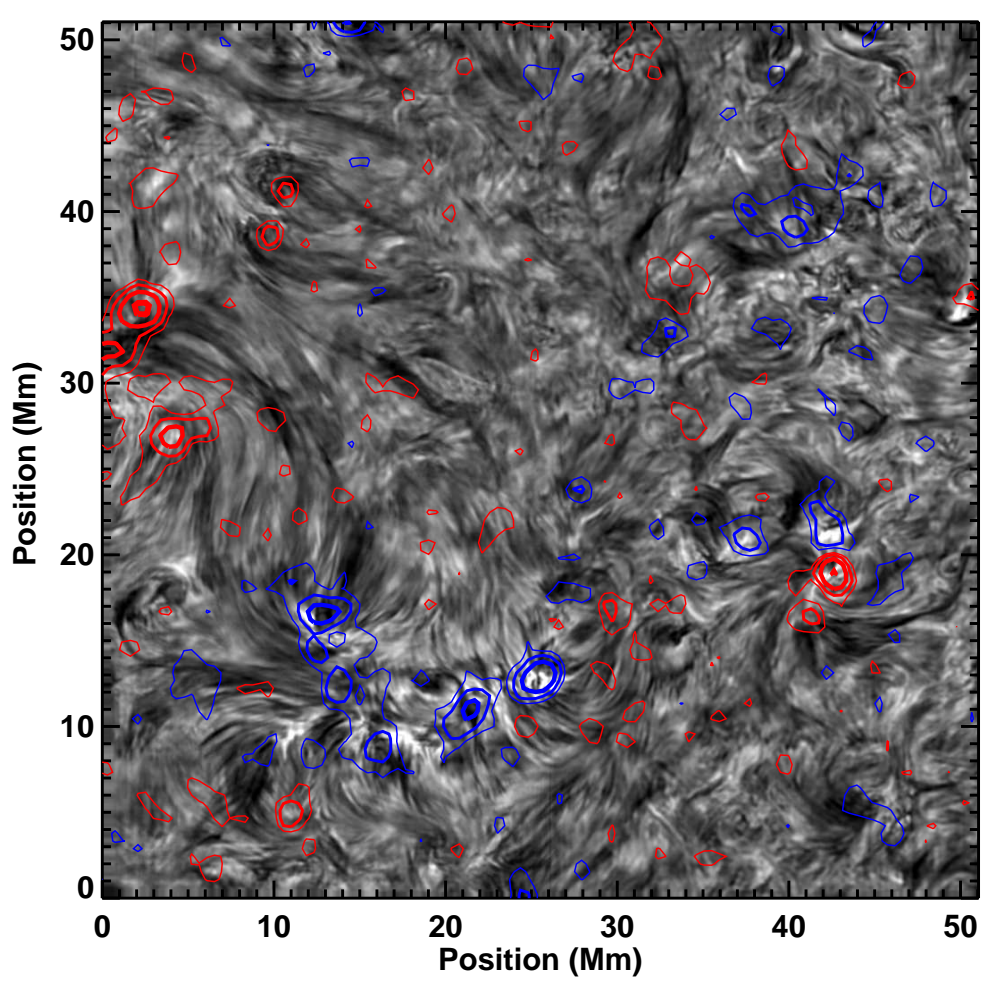

Fig. 2.- Full field-of-view NST H $\alpha$ image coaligned with the high-resolution MDI magnetogram (red contours for $+10,+20,+40 \mathrm{G}$, and blue oness for $-10,-20,-40 \mathrm{G}$ ).

the velocity field profile is affine, whereas the better-known technique of local correlation tracking assumes that the velocity field is uniform (Chae \& Sakurai 2008). The errors of the NAVE tracking using real data were investigated in detail by Chae \& Moon (2009).

As shown in Figure 1 we extracted magnetic information from full-disk magnetograms of 96 minutes cadence and one high-resolution magnetogram of 1 minute cadence taken by Michelson Doppler Imager (MDI) onboard Solar and Heliospheric Observatory (SOHO). The high-resolution magnetogram is the only magnetogram taken by MDI during the period of our observation, and regrettably, no magnetograms were taken by the Solar Optical Telescope onboard Hinode at that time. The alignment of the high-resolution magnetogram and the $\mathrm{H} \alpha$ images was done using network features commonly identified in the two kinds of data (see Figure 2).

The well-resolved morphology of the fine-scale elongated structures seen in the NST $\mathrm{H} \alpha$ images allowed us to infer the field-line connectivity of the magnetic fragments without ambiguity. Specifically, we identified from the $\mathrm{H} \alpha$ images the footpoints, directions, and connections of fibrils and loops, and inferred from the high-resolution MDI magnetogram 
the polarity of each footpoint. This procedure provided us information abundant enough for us to group the fibrils and loops depending on the connectivity and to draw the curves separating between different groups fairly well. These curves correspond to the intersection of separatrices and the solar surface, where a separatrix is a surface separating two magnetic domains. Note that this kind of analysis is mostly based on the $\mathrm{H} \alpha$ morphology, and little on the detailed magnetic structures seen in the magnetogram because its spatial resolution $\left(\geq 1.2^{\prime \prime}\right)$ is much poorer than that of the NST H $\alpha$ image $\left(<0.2^{\prime \prime}\right)$, and the Gaussian smoothing applied the magnetogram with a FWHM of 2 pixels to suppress noise further deteriorates its spatial resolution. Moreover, it is known that one-minute cadence MDI magnetograms like the one used for our analysis are subject to fluctuations of solar oscillation origin that may bring about random spatial offsets of a significant fraction of a pixel in magnetic concentrations (e.g., Chae \& Moon 2009).

\section{RESULTS}

During the observations the Sun was very quiet, without having had any spot for several months. As can be seen from the left of Figure 1, the observed region is truly representative of the quiet Sun, and the CMF is nothing more than one of the ordinary CMFs that are commonly found in the quiet Sun. The right of Figure 1 shows that our observations covered only a short period at the later phase of flux cancellation that proceeded for longer than 8 hours. The rate of flux cancellation in this CMF was 2 to $3 \times 10^{18} \mathrm{G} \mathrm{cm}^{2} \mathrm{~h}^{-1}$, which is typical of CMFs determined using SOHO/MDI (Park et al. 2009).

In the high-resolution NST $\mathrm{H} \alpha$ image of the region as presented in Figure 3, we see a multitude of thin fibrils and loops. They have widths in the range from 0.20 to $0.35^{\prime \prime}$ or from $150 \mathrm{~km}$ to $260 \mathrm{~km}$. The swirling pattern of the fibrils around the positive pole indicates that the magnetic field configuration is far from a potential one. As a matter of fact, we found it impossible for us to simulate this pattern and other patterns of fibrils and loops by extrapolating the photospheric magnetic field to the chromosphere even assuming a linearforce-free field, not to speak of a potential field. Note that our drawing of the two curves in Figure 3 is not based on the field extrapolation, but based on the morphology of fibrils and loops seen in the $\mathrm{H} \alpha$ images. These two curves divide the region into four domains inside each of which all the field lines have the same connectivity.

The field lines in domain III start from the magnetic fragment of positive polarity, but are not connected to the other magnetic fragment of opposite polarity constituting the CMF. Moreover, we found that these highly twisted field lines cannot be modelled by a force-free field unless the negative magnetic fragment is removed. Therefore, we regard 


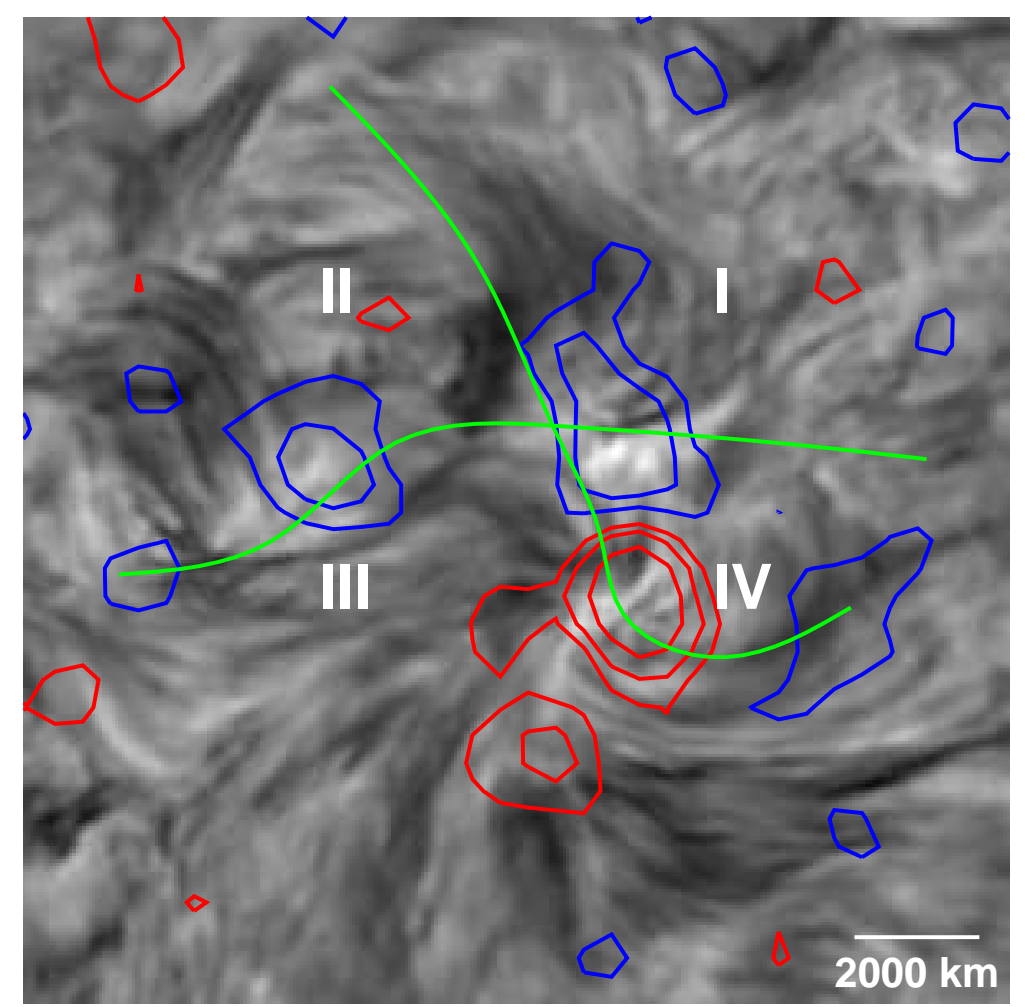

Fig. 3.- NST H $\alpha$ centerline image of the region of $22^{\prime \prime} \times 22^{\prime \prime}(16000 \mathrm{~km} \times 16000 \mathrm{~km})$. The two green curves represent the intersections of two separatrics and the surface.

domain III as a pre-reconnection domain independent of the negative magnetic fragment. Similarly, field lines in domain I start from the negative fragment, but are not connected to the positive fragment so that domain I is a pre-reconnection domain independent of the positive magnetic fragment. On the other hand, the field lines in domain IV connect the two magnetic fragments, so domain IV may be a post-reconnection domain. Domain II may be another post-reconnection domain, in which all the field lines are connected to remote areas outside the CMF.

It is likely that magnetic reconnection occurred somewhere along the separator, the intersection of the two separatrices. The separator is a curve in the three-dimensional space, but its intersection with the solar surface appears as a null point. This point is located outside the magnetic fragment of negative polarity, as is often found in the theoretical models of magnetic field topology (e.g., Gorbachev \& Somov 1989; Restante et al. 2009).

It is theoretically expected that plasma in the post-reconnection domains should be either heated and/or accelerated as a result of the release of magnetic energy during magnetic reconnection. As a matter of fact, an examination of temporal variation presented in Figure 4 


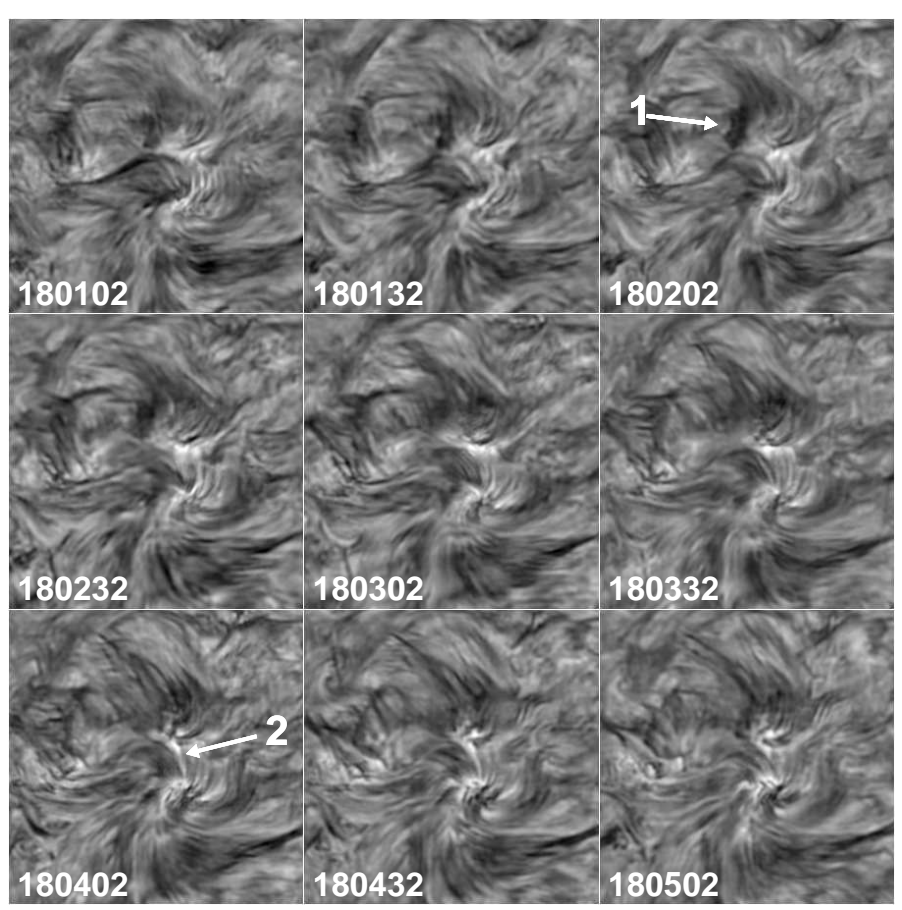

Fig. 4. - Temporal variation of the CMF region as seen in the NST H $\alpha$ images. The dark cloud is numbered 1 , and the bright loop, 2 . The field of view shown here is $22^{\prime \prime} \times 22^{\prime \prime}(16000$ $\mathrm{km} \times 16000 \mathrm{~km})$.

reveals a couple of features that are quite consistent with these expectations. The first one is the ejection of a dark cloud in the post-reconnection domain II. This cloud appeared near the separator, reached its highest contrast of 30\% around 18:01:32 UT, and then moved away from it at a speed of $9 \mathrm{~km} \mathrm{~s}^{-1}$ as indicated in Figure 5. The cloud expanded as it moved obliquely upward, with its contrast decreasing with time. At the time of its highest visibility (18:02:02 UT), its size was $1000 \mathrm{~km}$ by $2000 \mathrm{~km}$. It became indiscernible 1.5 minute later (after 18:03:32 UT).

The next event attracting our interest is the appearance of a tiny bright loop in domain IV as seen in Figure 4. This is the latest one of several loops of similar kind visible in the figure. At the time of its peak development (18:04 UT), the loop was $2200 \mathrm{~km}$ long and $210 \mathrm{~km}$ wide. Its brightest part is the northern footpoint that was $2000 \mathrm{~km}$ away from the starting point of the dark cloud on the plane of the sky. Here the intensity increased from 1.4 to the peak of 2.1 in about 1.3 minutes and then decreased down to 1.4 in 2.5 minutes as plotted in Figure 5. The brightening also occurred in the other footpoint of the loop, even though it was not so conspicuous as the northern footpoint. The cross-correlation analysis of the two intensity profiles indicates that the brightening of the southern footpoint lags behind 

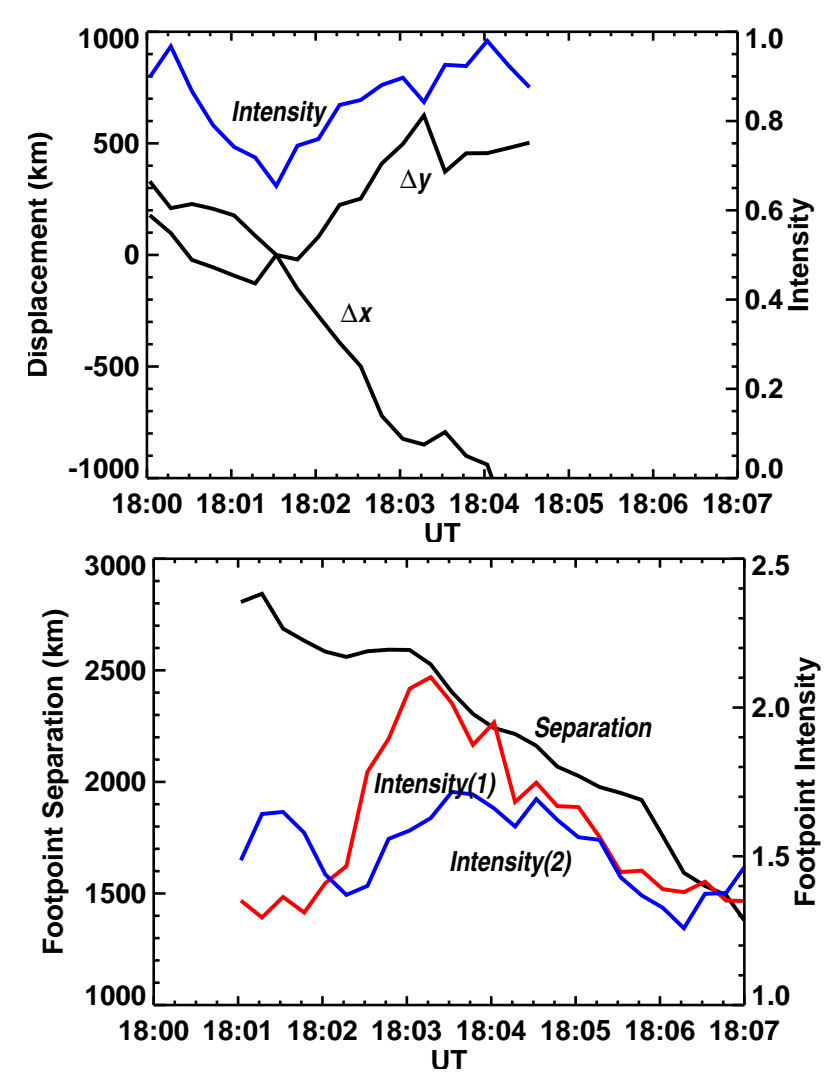

Fig. 5.- Top: temporal variations of intensity and displacement of the dark cloud in magnetic Domain II. Bottom: variations of intensities and separation of two footpoints of the bright loop in magnetic domain IV.

the northern one by $23 \mathrm{~s}$. It is noteworthy that the brightening of the loop occurred after the ejection of the dark cloud. There is a time difference of 1.8 minute between the time of the minimum brightness of the dark cloud and that of the maximum brightness of the loop.

An interesting and noteworthy characteristic of the bright loop is that it was shrinking. This shrinkage took place after the peak of brightening as well as after the ejection of the dark cloud. The separation between the two footpoint decreased from $2580 \mathrm{~km}$ at 18:03 UT to $1760 \mathrm{~km}$ at 18:06 UT, implying that the two footpoints approached each other at the relative speed of $4.6 \mathrm{~km} \mathrm{~s}^{-1}$. 


\section{DISCUSSION}

The observed features - the division of magnetic domains, the ejection of a small dark cloud, and the appearance of the tiny bright loop - support the notion that magnetic reconnection was occurring somewhere in the chromosphere. The dark cloud may correspond to either reconnection outflow or a plasmoid that was ejected from the current sheet and moved obliquely upward. It appeared in absorption because the density is so low that collisional processes are negligible, and scattering dominates the radiative process. On the other hand, the bright loop may represent the post-reconnection loop that was formed by reconnection, and moved down. This loop is the evidence that heating occurred. The reason why it appeared in emission in contrast to the dark cloud is that it may have not only excess temperature, but also high density so that collisional processes play a role. This loop may be regarded as the smallest miniature of a post-flare loop that was ever observed so far. The key point is that at the middle of the dark cloud and the bright loop was the intersection of the separator with the surface, which strongly suggests that these are the consequence of magnetic reconnection that occurred on the separator.

Our observations provide strong evidence that reconnection takes place well inside the chromosphere. One unique feature of our observations is that the size of the magnetic system involved in the magnetic reconnection is small - about $2000 \mathrm{~km}$, as inferred from either the footpoint separation of the bright loop or the plane-of-sky distance between the bright loop and the dark cloud. Even though it's not easy to infer the altitude of the atmospheric level where reconnection occurred, we guess that it should be of the order of half of the system size: about $1000 \mathrm{~km}$.

Our observations significantly improve the understanding on how reconnection in the chromosphere is related to the cancellation of flux observed in the photosphere. The important finding is a shrinking bright loop. It is well known from observations (e.g., Chae et al. 2002b) and MHD simulations (e.g., Dreher et al. 1997; von Rekowski et al. 2006) that the converging motion of two magnetic fragments is a potential driver of flux cancellation and magnetic reconnection. The same kind of converging motion is identified in the right top of Figure 1. The rapid convergence of two footpoints we found, however, seems to be distinct from this well-known converging motion of magnetic fragments for several reasons. Firstly, the length scales of measurement are different. The present measurement of the motion was done when the separation is less than $3000 \mathrm{~km}$, but previous measurements of the conventional converging motion using MDI magnetograms were confined to the separations greater than $3000 \mathrm{~km}$ (Litvinenko et al. 2007) because of the limitation of spatial resolution. Secondly, the value of the converging speed is different. Here we obtained $4.6 \mathrm{~km} \mathrm{~s}^{-1}$. This is much higher than the typical relative speed of $0.6 \mathrm{~km} \mathrm{~s}^{-1}$, (Litvinenko et al. 2007). Most 
of all, the shrinking represents a rapid convergence of two footpoints that are magnetically connected by a loop, whereas it is known that two magnetic fragments approaching and cancelling each other are not magnetically connected to each other initially (Wang \& Shi 1993). In addition, the conventional converging motion of magnetic fragments usually persists for several hours, but the shrinking motion lasted for a short period of about ten minutes after the ejection of dark cloud and the peak of brightening.

We regard the shrinking as strong and direct evidence for the long-standing notion that magnetic reconnection occurring above the surface creates new sets of fieldlines that are concave downward and subsequently submerge below the surface (Zwaan 1987). What is surprising is that the initial footpoint separation, $2800 \mathrm{~km}$, is not smaller than the critical maximum values of the footpoint separation theoretically proposed for a loop to submerge by overcoming magnetic buoyancy. Based on a simple comparison of magnetic tension force and magnetic buoyancy, van Ballegooijen \& Martens (1989) found a critical footpoint separation of $4 H_{s}$ where $H_{s}$ is the hydrostatic pressure scale height. A higher value of $2 \pi H_{s}$ came from Parker (1979) who considered the equilibrium path of a flux tube, which we estimate to be about $1500 \mathrm{~km}$ in the chromospheric condition. Our speculation is that the downward momentum produced by reconnection may make it easier for the post-reconnection loop to submerge than a loop initially in equilibrium. This means that reconnection leading to the cancellation of flux can in the photosphere can occur higher than previously thought. Our observations suggest that it takes an order of 10 minutes for the fieldlines produced by the reconnection at that altitude to extract down and submerge below the surface. It will be important to properly understand the dynamics of downward transport of magnetic flux after reconnection based on further observational results. Particularly necessary is to determine the converging speed of magnetic fragments at a few tenth of arc second resolution, to compare with the high converging speed of the footpoint we observed in the high resolution $\mathrm{H} \alpha$ images.

Moreover, our finding of moving dark clouds and tiny bright loops is observational evidence that reconnection is not a steady and continuous process, but consists of a series of discrete events in each of which one plasmoid-like cloud and one loop may be formed. A detailed investigation of this aspect will greatly improve our understanding of physics underlying magnetic reconnection in the chromosphere.

We appreciate the referee's critical comments, which were helpful in our improving the manuscript. This work was supported by the National Research Foundation of Korea (KRF2008-220-C00022), NSF (ATM-0745748 and ATM-0847126), NASA (NNX08BA22G) and AFOSR (FA 9550-09-1-0655). 


\section{REFERENCES}

Chae, J., Choi, B.-K., \& Park, M.-J. 2002, Journal of Korean Astronomical Society, 35, 59

Chae, J., Moon, Y.-J., Wang, H., \& Yun, H. S. 2002, Sol. Phys., 207, 73

Chae, J., Moon, Y.-J., \& Pevtsov, A. A. 2004, ApJ, 602, L65

Chae, J., \& Sakurai, T. 2008, ApJ, 689, 593

Chae, J., \& Moon, Y. J. 2009, J. Korean. Astron. Soc., 42, 61

Dreher, J., Birk, G. T., \& Neukirch, T. 1997, A\&A, 323, 593

Foukal, P. 1971, Sol. Phys., 20, 298

Goode, P. R., Denker, C. J., Didkovsky, L. I., Kuhn, J. R., \& Wang, H. 2003, Journal of Korean Astronomical Society, 36, 125

Goode, P. R., Coulter, R., Gorceix, N., \& Cao, W. 2010, Astron. Nach., in press

Gorbachev, V. S., \& Somov, B. V. 1989, SvA, 33,, 57

Harvey, K. L., Jones, H. P., Schrijver, C. J., \& Penn, M. J. 1999, Sol. Phys., 190, 35

Kubo, M., \& Shimizu, T. 2007, ApJ, 671, 990

Litvinenko, Y. E. 1999, ApJ, 515, 435

Litvinenko, Y. E., Chae, J., \& Park, S.-Y. 2007, ApJ, 662, 1302

Litvinenko, Y. E., \& Chae, J. 2009, A\&A, 495, 953

Livi, S. H. B., Wang, J., \& Martin, S. F. 1985, Australian Journal of Physics, 38, 855

Martin, S. F., Livi, S. H. B., \& Wang, J. 1985, Australian Journal of Physics, 38, 929

Park, S., Chae, J., \& Litvinenko, Y. E. 2009, ApJ, 704, L71

Parker, E. N. 1979, Cosmical Magnetic Fields (Oxford: Clarendon Press)

Priest, E. R., \& Forbes, T. G., Magnetic Reconnection, Cambridge Univ. press, Cambridge)

Restante, A. L., Aulanier, G., \& Parnell, C. E. 2009, å, 508, 433

van Ballegooijen, A. A., \& Martens, P. C. H. 1989, ApJ, 343, 971 
von Rekowski, B., Parnell, C. E., \& Priest, E. R. 2006, MNRAS, 369, 43

Wang, J., \& Shi, Z. 1993, Sol. Phys., 143, 119

Wöger, F., von der Lühe, O., \& Reardon, K. 2008, A\&A, 488, 375

Zwaan, C. 1987, ARA\&A, 25, 83 